\title{
Establishing a face-to-face dermatology clinic during the COVID-19 pandemic; supporting hospital staff
}

\author{
Authors: Sofia Hadjieconomou, ${ }^{A}$ Kamath Sandeep ${ }^{B}$ and Jenny Hughes ${ }^{B}$
}

\section{Introduction}

The COVID-19 pandemic forced changes to the daily work routine of hospital staff (HS) around the world. HS faced skin problems caused or worsened by the use of personal protective equipment (PPE), but also by the rigorous hand hygiene regimen.

\section{Materials and methods}

A dermatology clinic that took place twice weekly and was led by dermatologists, wearing appropriate PPE, was commenced to treat skin problems affecting all HS during the COVID-19 pandemic. We conducted a prospective observational case series from a single district general hospital in Wales, UK. Over a 3-month period, a total of $100 \mathrm{HS}$ (81 female and 19 male) working in COVID-19 and non-COVID-19 areas of the hospital attended the clinic and were all included as participants. Data comprised demographic data, current occupation, duration of symptoms, past medical history, regular medication and treatment interventions.

\section{Results and discussion}

Irritant hand dermatitis was the most common diagnosis, present in $52 \%$ of attendees. Mask-related adverse effects were seen in $7 \%$ and worsening of pre-existing skin conditions in $42 \%$. Dermatological conditions unrelated to PPE use were seen in $25 \%$ of patients, and $14 \%$ of patients mentioned that emotional stress worsened their skin disease. Irritant hand dermatitis was treated in accordance with Zhang et al, ${ }^{1}$ with a lighter emollient for day/work use and a greasy emollient for home use. Inflamed skin was treated with topical steroids and soap substitute use was proposed only at home, as it has not been validated for eradication of SARS-CoV-2. Pressurerelated facial symptoms did not present in as high percentages as reported by Jiang et al, ${ }^{2}$ who only included staff treating COVID-19 patients. Additionally, the hospital had already instituted advice for skin protectant film use prior to mask application and avoidance of mask over-tightening. We treated pressure-related facial symptoms with a combination of barrier cream usage and hydrocolloid dressings applied over pressure points. A repeat mask-fit test, with the dressings already applied, was required to ensure an effective seal. Exacerbations of pre-existing skin conditions were managed following standard treatment of care.

\section{Conclusion}

To our knowledge, this is the first face-to-face dermatology clinic for HS established during the COVID-19 pandemic. We recognise that the sample size was small and data were collected from a single centre; however, all diagnoses were made by a specialist, and HS from all occupations were included in the study. As a result of the clinic, there was an unintended benefit of supporting staff wellbeing during the pandemic.

\section{Conflicts of interest}

None declared.

\section{References}

1 Zhang B, Zhai R, Ma L. 2019 novel coronavirus disease epidemic: skin protection for healthcare workers must not be ignored. J Eur Acad Dermatol Venereol 2020;34:e434-5.

2 Jiang Q, Song S, Zhou J et al. The prevalence, characteristics, and prevention status of skin injury caused by personal protective equipment among medical staff in fighting COVID-19: a multicenter, cross-sectional study. Adv Wound Care 2020;9:357-64. 